\title{
Effet captivant et apaisant de la médiation par tablette au musée : mesures physiologiques et motivationnelles
}

The captivating and soothing effect of tablets in museums: physiological and

motivational measurements

Fanny Bougenies, Sylvie Leleu-Merviel et Laurent Sparrow

\section{CpenEdition}

Journals

Édition électronique

URL : http://journals.openedition.org/edc/6565

DOl : 10.4000/edc.6565

ISSN : 2101-0366

Éditeur

Université Lille-3

\section{Édition imprimée}

Date de publication : 1 juin 2016

Pagination : 87-108

ISBN : 978-2-917562-15-4

ISSN : $1270-6841$

\section{Référence électronique}

Fanny Bougenies, Sylvie Leleu-Merviel et Laurent Sparrow, «Effet captivant et apaisant de la médiation par tablette au musée: mesures physiologiques et motivationnelles », Études de communication [En ligne], 46 | 2016, mis en ligne le 01 juin 2018, consulté le 05 mai 2019. URL : http:// journals.openedition.org/edc/6565; DOI : 10.4000/edc.6565 


\section{Effet captivant et apaisant de la} médiation par tablette au musée:

mesures physiologiques et motivationnelles

The captivating and soothing effect of tablets in museums: physiological and motivational measurements

Fanny Bougenies

Univ Lille Nord de France, F-59000, Lille, France UVHC, DeVisu, F-59313, Valenciennes, France fannybou@hotmail.com

Sylvie Leleu-Merviel

Univ Lille Nord de France, F-59000, Lille, France UVHC, DeVisu, F-59313, Valenciennes, France Sylvie.Merviel@univ-valenciennes.fr

Laurent Sparrow

Univ Lille Nord de France, F-59000, Lille, France Lille 3, Laboratoire SCALAB, F-59000, Lille, France laurent.sparrow@univ-lille3.fr 
Muséo+ PBA Lille est un dispositif de médiation muséale conçu pour permettre à tous les publics, notamment les enfants en situation de handicap, de bénéficier d'une visite guidée par tablette au musée. II a été testé grâce à un protocole original associant deux outils classiques de la psychologie cognitive : les mesures physiologiques (oculométriques et électrodermales) et les mesures émotionnelles via le diagramme valence-activation. Les résultats convergent et révèlent notamment que chaque expérience de visite (en termes de sensations, d'émotions et d'expériences vécues et/ou ressenties) est strictement personnelle. Ils montrent ainsi l'intérêt d'une recherche située au niveau de l'individu.

Mots-clés : médiation, musée, tablette, dispositif, conception universelle, évaluation.
Muséo+ PBA Lille is a mediation device designed to allow everyone, including children with disabilities, to enjoy a guided tour through museum spaces. It has been tested through an original protocol that combines two classic tools of cognitive psychology: physiological measures (eye tracking and electrodermal activity) and emotional measures via the valence-activation diagram. The results converge: they reveal that each particular visitor experience (in terms of sensations, emotions and lived and/or perceived experiences) is strictly personal. Results thus show the interest of research located at the individual level.

Keywords: mediation, museum space, digital tablet, device, design for all, assessment. 
L'accès au musée n'est pas aussi aisé pour tous. Il est notamment compliqué par un handicap physique, mental et/ou comportemental. Les questions d'accessibilité physique donnent lieu à des aménagements de l'espace architectural, par exemple pour garantir l'accès aux personnes à mobilité réduite. Mais on trouve aussi des difficultés liées à la communication (par exemple pour les publics sourds, car les guides maîtrisant la Langue des Signes FrançaiseLSF - ne sont pas nombreux), d'autres liées à la compréhension des œuvres en général (déficients intellectuels) ou d'autres encore qui éprouvent des difficultés lorsqu'ils se trouvent dans un espace public (autistes).

Muséo+ PBA Lille, dispositif de médiation muséale par tablette, a été conçu dans le but de contourner en partie ces obstacles avec l'objectif de permettre à tous les publics de bénéficier d'une visite au musée. II a été expérimenté au Palais des Beaux-Arts de Lille auprès de cent trente enfants relevant de cinq catégories: autistes, déficients intellectuels, sourds, précoces et sans handicap'. L'étude partielle présentée ici s'appuie sur un protocole original associant deux outils classiques de la psychologie cognitive: les mesures physiologiques (oculométriques et électrodermales) et les mesures émotionnelles via le diagramme valence-activation. L'objectif est de montrer comment une telle approche innove et renouvelle les perspectives concernant la communication au musée. Elle révèle notamment que chaque expérience de visite (en termes de sensations, d'émotions et d'expériences vécues et/ou ressenties) est strictement personnelle, non transposable à une autre personne. Dès lors, son analyse doit se situer au niveau de l'individu, et le recours aux statistiques a pour biais de moyenner des résultats incommensurables en termes d'expérience.

\section{1.}

\section{L'approche Design for all et sa mise en œuvre}

\subsection{Les grands principes de la conception universelle}

L'approche dite de design for all ou conception universelle (Houriez et al., 2013), consiste à intégrer un ou plusieurs handicaps à la conception d'un objet non pas spécifique à un public préalablement segmenté, mais destiné à tous. Comme le précise le Conseil de l'Europe, "le design for all est une stratégie qui vise à concevoir et à composer différents produits et environnements qui soient, autant que faire se peut et de la manière la plus indépendante et naturelle possible, accessibles, compréhensibles et utilisables par tous, sans devoir

1 Pour une description exhaustive du protocole complet, voir (Bougenies et al., 2015, paru dans l'intervalle). D'autres outils plus classiques ont également été mis en œuvre: questionnaires et entretiens auprès des accompagnants, observation ethnographique, captation vidéo, protocole VIAGE. C'est dans la mesure où cet article focalise sur les outils de la psychologie cognitive que cette étude est partielle. 
recourir à des solutions nécessitant une adaptation ou une conception spéciale» (ResAP, 2001). Ainsi, au lieu d'adapter l'existant aux personnes en situation de handicap, le design for all propose d'intégrer le handicap directement au cahier des charges pour concevoir pour tous différemment.

Le handicap devient par conséquent une source d'innovation et non une contrainte comme le prévoient les dispositions légales pour les établissements recevant du public, notamment les musées. Outre la capacité à créer du lien et du partage entre différentes communautés avec et sans handicap, la conception universelle permet de repenser la visite au musée pour tous en entrant dans une démarche conceptuelle ancrée sur la résolution de problème en amont. A titre d'exemples: les problèmes de motricité justifient l'utilisation du «drag and drop" et l'emplacement des boutons; la coque de l'Ipad est pourvue d'une poignée permettant la déambulation et la stabilité sur sol; la présentation du contenu informationnel est pensée de manière à convenir à d'autres visiteurs sans handicap en quête d'informations et/ou adeptes de la ludologie (public adolescent réticent à la découverte muséale).

La perspective adoptée ici focalise sur les difficultés communicationnelles liées à une diversité de besoins des personnes en situation de handicap sensoriel ou intellectuel. En effet, il existe à ce jour dans les musées très peu d'environnements qui restent opératoires en cas de déficit intellectuel léger ou profond, d'autisme avéré, de surdité partielle ou totale.

\subsection{L'application Muséo+}

Dans le prolongement de Muséo (Leleu-Merviel et Kounakou, 2011), I'application Muséo+ propose aux enfants entre six et douze ans une visite audio et visio-guidée par i-Pad au Palais des Beaux-Arts de Lille. Elle répond aux attendus de la conception universelle.

Les sept œuvres du parcours ont été choisies par les équipes chargées de la médiation et de l'accueil des publics au Palais des Beaux-Arts. L'association Signes de Sens, partenaire du projet, a effectué la conception du dispositif et en a supervisé la réalisation. L'application contient trente minutes de vidéo et dix jeux interactifs. Une comédienne y joue le rôle de Karine, jeune passionnée d'art qui prépare sa prochaine visite au musée. Un perturbateur espiègle en animation 2D, "Mange-Tout», désorganise tous ses dossiers. Karine requiert l'aide des enfants pour remettre de l'ordre.

Les enfants choisissent l'œuvre qu'ils veulent découvrir à partir du menu général. Un plan apparaît pour les aider à trouver l'œuvre dans les salles, puis l'explication et les jeux commencent. Si nécessaire, les enfants n'ont pas à 
découvrir toutes les œuvres pour pouvoir accéder à la vidéo de conclusion et de fin².

Le parcours dure entre quarante-cinq minutes et une heure selon le rythme des enfants et le nombre d'œuvres découvertes. Toutes les vidéos de l'application sont doublées en Langue des Signes Françaises (LSF), avec une voix-off et un sous-titrage.

Ainsi, à partir du parcours à accomplir en autonomie à l'intérieur-même du Palais des Beaux-Arts, les enfants apprennent à se repérer, à observer, à apprécier les œuvres à l'aide d'activités ludiques et de pastilles vidéo scénarisées servant de guide.

\subsection{Problématique d'évaluation du dispositif}

Muséo+ ambitionne de capter l'intérêt du visiteur et de maintenir cette attention tout au long de l'expérience afin qu'il soit dans un état propice à la découverte, à l'acquisition éventuelle de connaissances nouvelles, ou plus simplement qu'il éprouve des émotions positives tout en se cultivant. Cependant, organiser le recueil de données concernant l'expérience de visite de tels publics $^{3}$, très peu communicants par définition, est un véritable défi (Kounakou et Verclytte, 2011).

(Bougenies et al., 2015) présente en détail le protocole inédit visant à effectuer l'évaluation complète du dispositif. II permet de recueillir des données concernant l'expérience de visite auprès des enfants eux-mêmes et interroge également les accompagnants. Il a été expérimenté auprès de cent trente enfants relevant de cinq catégories: autistes, déficients intellectuels, sourds, précoces et sans handicap ${ }^{4}$. Composé de plusieurs outils et méthodes croisés, l'ensemble répond à une évaluation pour tous.

Le présent article focalise sur une expérimentation exploratoire qui constitue une composante particulière du protocole complet. Il s'agit d'une méthode ne requérant pas de verbalisation et permettant de mesurer, en temps réel, les réactions d'un visiteur afin d'estimer son niveau de concentration ainsi que ses réactions émotionnelles et son appréciation. En ayant recours notamment aux indicateurs dont la pertinence est acquise par les travaux de la psychologie cognitive, cette méthode tente de réduire la part de subjectivité que comporte

2 Une présentation vidéographique de Muséo+ est accessible à l'adresse http:// www.signesdesens.org/nos-realisations/museo-v2/ (consulté le 13septembre2015).

3 Beaucoup d'entre eux sont de fait dans une incapacité partielle ou totale à communiquer verbalement.

4 C'est l'association Signes de Sens, spécialisée dans la médiation pour l'enfant en situation de handicap, qui a organisé l'expérimentation, et effectué le recrutement auprès d'une part des familles, d'autre part des Instituts Médicalisés pour l'Enfance - IME- de son carnet d'adresses. 
la verbalisation en la croisant avec des mesures. Elle associe des relevés physiologiques suivant une approche expérimentale relevant de la psychologie cognitive, et des appréciations déclaratives exprimées graphiquement.

\section{2.}

\section{Mesures physiologiques}

\subsection{Principes des mesures oculométriques et électrodermales}

En appliquant les théories de la psychologie cognitive, il est possible de repérer, à l'aide d'indicateurs physiologiques et comportementaux, l'état psychologique d'une personne, les émotions qu'elle peut ressentir, son niveau de concentration, etc. En effet, des indicateurs comme l'évolution du diamètre pupillaire et la taille des saccades oculaires renseignent sur l'état de concentration du participant (Steinhauer et al., 2004). Dans une situation comme celle qui nous intéresse, on observe deux types différents de comportements: le comportement d'exploration et le comportement d'exploitation (Gilzenrat et al., 2010).

La phase d'exploration est le moment où l'observateur explore son environnement visuel. Cette phase se caractérise par un nombre plus important de saccades de grandes amplitudes immédiatement suivies par des fixations (moment où l'œil est stable) longues. La direction de ces saccades est, au moins dans un premier temps, sous le contrôle de la saillance visuelle (Land, Mennie et Rusted, 1999). On observe aussi un niveau tonique (i.e. niveau de base, constant) d'activation assez faible, ce qui conduit à l'observation de réponses phasiques (i.e. réponses déclenchées en réaction à l'environnement externe, elles se superposent au niveau tonique) pupillaires plus importantes (Gilzenrat et al., 2010). Ces réponses phasiques pupillaires, si elles sont absentes, indiquent que l'exploration n'est pas réalisée correctement. Dans ce cas, il convient de vérifier si la saillance visuelle que constitue l'interface présente sur la tablette est assez élevée. Les réponses phasiques pupillaires indiquent que l'attention sélective est mobilisée, ce qui favorise ensuite, entre autres choses, la mémorisation (Papesh, Goldinger et Hout, 2012).

Le deuxième type de comportement, l'exploitation, est une phase pendant laquelle le participant utilise les données récoltées dans la phase d'exploration pour interagir avec son environnement. Cette phase est caractérisée par un nombre plus important de saccades oculaires de petites tailles (le regard reste dirigé dans une zone qui équivaut à la zone fovéale, soit deux à trois degrés). Les fixations qui suivent sont plus courtes. Au fur et à mesure que l'engagement du participant augmente, on observe une augmentation du niveau tonique du diamètre pupillaire. Les réactions phasiques seront plus nombreuses mais de plus faible amplitude. Cette configuration témoigne d'une implication plus importante de la cognition, en particulier de l'attention sélective. Si un tel 
comportement n'est pas observé, c'est que, probablement, le participant n'a pas réussi à prendre correctement en main l'interface.

En plus de ces données oculométriques, des données physiologiques permettant d'affiner l'analyse peuvent aussi être enregistrées à l'aide d'un bracelet doté de capteurs biologiques permettant d'enregistrer l'activité électrodermale de la peau et les mouvements. L'activité électrodermale est sous le contrôle du système nerveux sympathique: ce réseau neuronal pilote le niveau global d'activation de l'organisme, c'est-à-dire le niveau de tonicité de l'activation nerveuse (Boucsein, 2012). Si nous observons une augmentation de la tonicité à la fois pour le diamètre pupillaire et pour l'activité électrodermale, alors on pourra considérer que le système nerveux sympathique est impliqué. Même s'il est primordial qu'une certaine tonicité soit apparente lorsqu'on interagit avec notre environnement, il semble plus intéressant de faire en sorte que le participant soit actif, engagé dans son action de découverte artistique. Or, cet engagement dépend plus de la cognition que du système nerveux sympathique. Et la cognition n'intervient que très peu sur la tonicité (en particulier de l'activité électrodermale) mais contrôle les réactions phasiques du diamètre pupillaire. Par conséquent, s'il est observé une augmentation de la fréquence des réactions phasiques du diamètre pupillaire, en l'absence d'activité électrodermale particulière, alors on pourra conclure que la cognition est impliquée. $\mathrm{Si}$, en revanche, l'activité électrodermale s'accroît alors que l'activité pupillaire reste stable, cela signera alors que l'activation du système sympathique est prépondérante et donc, qu'il s'agit d'une réaction émotionnelle (Reinhardt, 2012).

Nous proposons donc d'étudier ces différentes hypothèses à l'aide d'un matériel ambulatoire permettant d'enregistrer les déplacements oculaires en mobilité (lunettes SMI ETG) couplé à un bracelet permettant d'enregistrer, toujours en mobilité, l'activité électrodermale (QSensor, Affectiva). Un exemple de tracé est présenté figure1. La courbe superposée traçant l'activité électrodermale (désormais dénommée AED) sur l'ensemble de la séance montre qu'après avoir subi une période de stress et d'excitation élevée en début d'utilisation, l'utilisateur, grâce à l'application Muséo+, parvient ensuite à se concentrer. On voit clairement sur la courbe que le niveau de stress diminue. Il est alors inféré que cet effet d'apaisement, conjoint avec la concentration de l'attention, produit par la médiation à l'aide de la tablette, a permis à l'utilisateur de pleinement bénéficier de sa visite (ce que confirment les enquêtes menées en parallèle). 


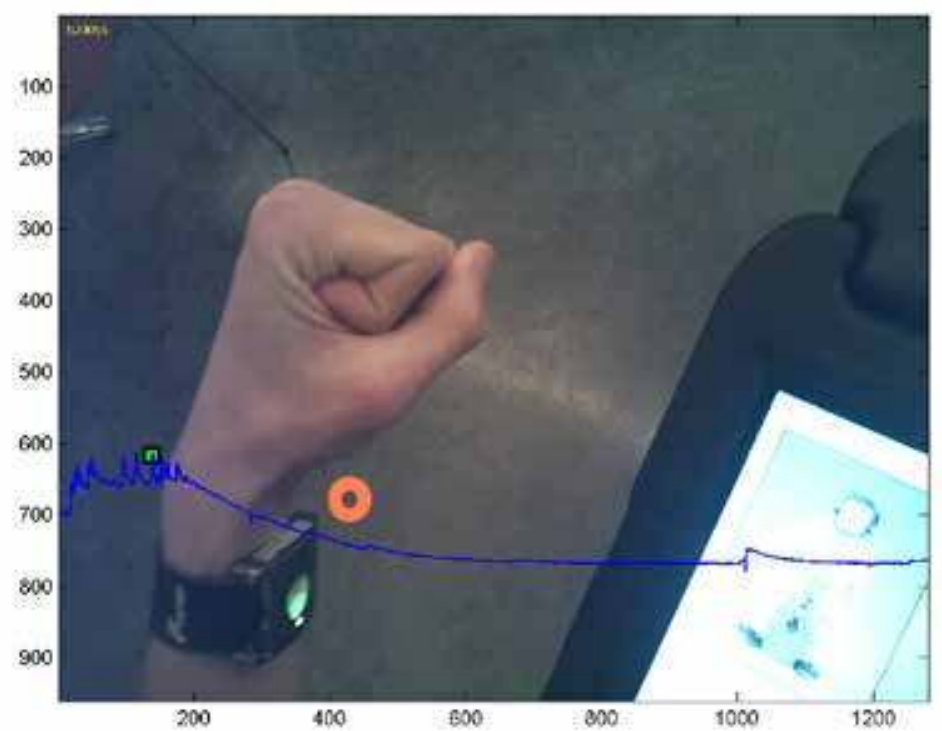

Figure 1: enregistrement couplé des déplacements oculaires et de l'activité électrodermale d'un enfant visitant un musée à l'aide de l'application Muséo+. L'image représente le point de vue de l'utilisateur et la position de son regard (rond clair). L'activité électrodermale est représentée par la courbe superposée, et le carré indique le niveau d'activité électrodermale instantané (caractérisé sur cette image par une activité tonique élevée sur laquelle de nombreux pics viennent se superposer, ce qui témoigne d'un niveau de stress élevé). Cette activité est enregistrée à l'aide du bracelet QSENSOR

(Affectiva) utilisé pour les mesures de l'AED, bracelet visible sur cette photo.

\subsection{Expérimentations effectuées}

La découverte par la médiation de Muséo+ comprend sept séquences vidéoludiques, entrecoupées par des phases de localisation de l'œuvre à chercher dans le musée, ici intitulées phases «plan». Nous avons procédé à sept enregistrements où l'enfant était équipé de lunettes oculométriques et du bracelet permettant d'enregistrer l'AED. Malheureusement, seuls quatre de ces enregistrements se sont avérés exploitables (voir tableau1). 


\begin{tabular}{ccc} 
Participant & code & diagnostic \\
\hline 1 & t2_Ed & TED \\
2 & t3_Ni & TED (non diag) \\
3 & t5_Gu & SH \\
4 & t6_Ma & SH \\
5 & t7_Ta & DI \\
6 & t8_Di & DI \\
7 & t4_Fi & SH
\end{tabular}

Tableau 1: liste des participants. Seuls les quatre premiers ont fait l'objet d'un enregistrement complet. Les participants cinq et six n'ont pas voulu/pu utiliser la tablette (voir ci-dessous) et le participant sept n'a pas pu chausser les lunettes (trop petit, les lunettes ne tenaient pas).

Pour deux enfants déficients intellectuels, les mesures n'ont pu être réalisées (participants cinq et six). Le premier n'a pas supporté d'être séparé de son groupe d'amis qu'il cherchait constamment du regard (figure 2, image de gauche) et a interrompu le test au bout de neuf minutes, tout en poursuivant le parcours avec ses amis.
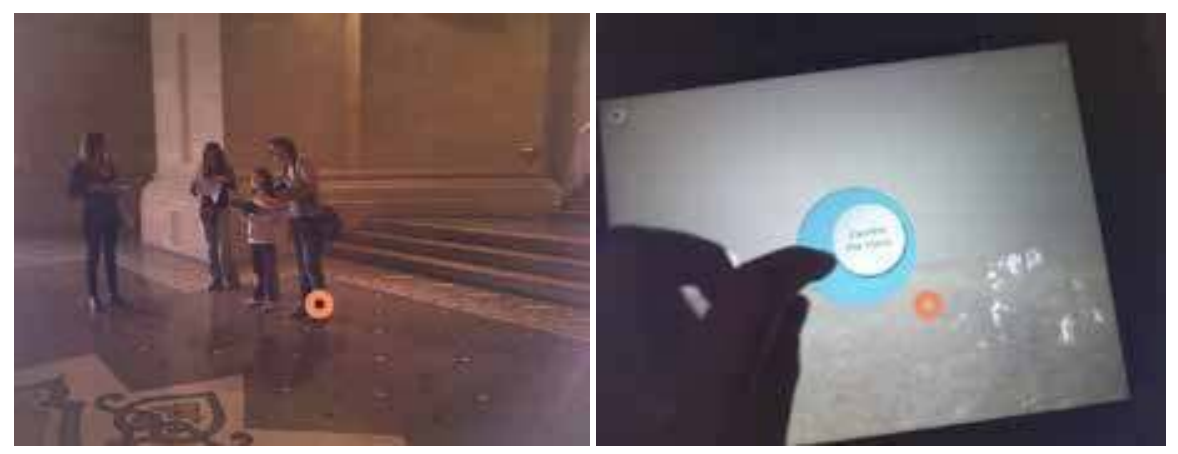

Figure 2: le participant $n^{\circ} 5$ n'a pas supporté être séparé de son groupe qu'il cherchait du regard constamment (image de gauche). Il a interrompu le test au bout de 9 minutes. Le deuxième (participant $n^{\circ} 6$ ) semble avoir rencontré des problèmes liés à l'utilisation de la tablette. Malgré le fait que des activités d'entraînement aient été proposées, l'enfant n'a pas réussi à utiliser l'application. 
Nous avons, dans un premier temps, analysé les durées de chacune de ces étapes (figure 3). Certaines sont plus courtes que d'autres (plan 3, 5 et 7) car l'œuvre suivante à découvrir se situait à proximité de l'endroit où se trouvait I'utilisateur. D'autres étapes sont plus longues (séquences 1, 3, 4 et 7; plan 6) mais globalement, les durées de réalisation de chacune de ces étapes sont remarquablement constantes d'un participant à l'autre.

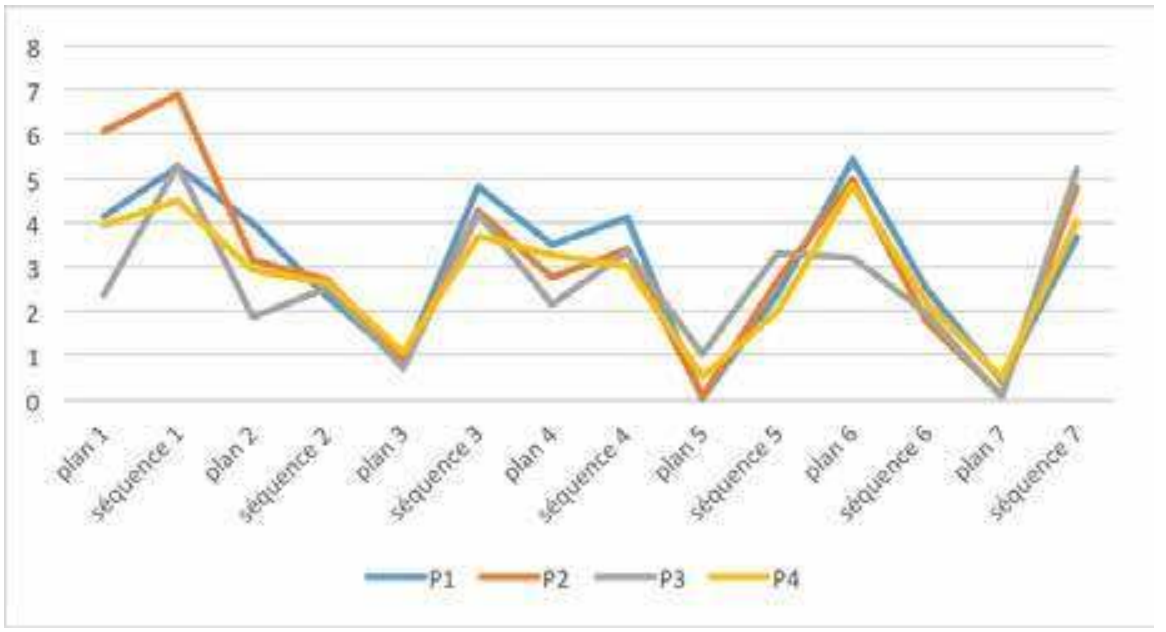

Figure 3: durée de réalisation de chacune des étapes de l'application Muséo+ pour les 4 participants ayant réalisé un test complet

\subsection{Résultats}

\subsubsection{Participant 1}

Le premier participant est un jeune enfant de 15 ans diagnostiqué comme autiste. Il est suivi depuis de nombreuses années et son entourage nous a prévenus qu'il souffrait aussi de phobie sociale, ce qui est courant dans les cas d'autisme.

Les tracés obtenus (figure 4) montrent une très forte activation sympathique: le nombre de pics est très élevé en tout début de séance et le niveau de tonicité est au maximum. Parallèlement, la part d'exploitation est faible. Le participant est, en début d'expérience, en état de stress très prononcé. La nouveauté de la situation (utilisation d'une tablette) et l'idée de devoir parcourir les allées du musée perturbent considérablement l'enfant. Une fois cette période d'adaptation terminée, l'enfant retrouve un niveau d'activation sympathique compatible avec une activité ludo-éducative, ce qui est confirmé par le tracé concernant la part d'exploitation (figure 4): elle augmente progressivement à partir de la séquence2, ce qui indique que l'utilisateur de la tablette se concentre sur les activités proposées. L'enfant reste ensuite dans le même niveau d'engagement jusqu'aux séquences 5 et 6 où l'on observe une part d'exploitation 
plus importante. Conjointement, les pics d'AED augmentent (surtout pour la séquence 6) mais comme le niveau tonique reste stable, on peut considérer qu'il ne s'agit pas ici d'une nouvelle montée de stress. L'enfant réagit à son environnement (augmentation des pics phasiques de l'AED), s'engage dans les activités proposées (augmentation de la part d'exploitation) et les symptômes d'une phobie sociale ne sont plus apparents.

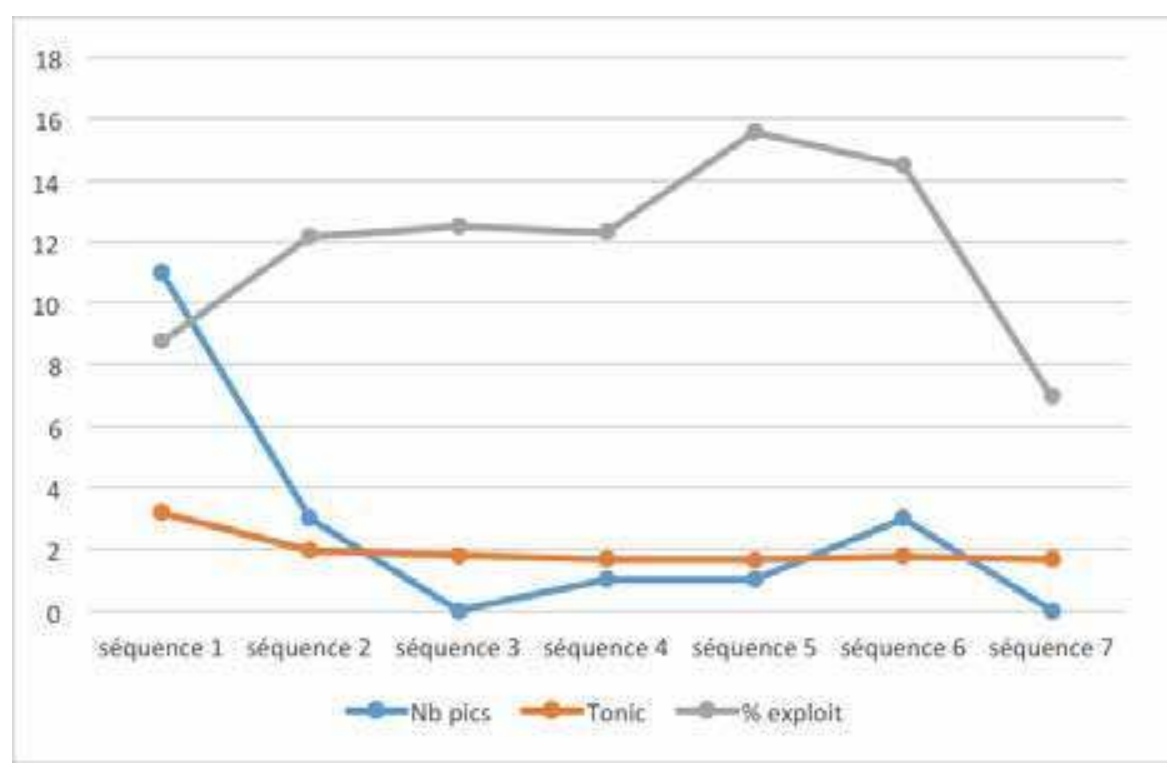

Figure 4: nombre de pics et niveau tonique de l'AED. Part d'exploitation oculaire pour le participant 1

On peut donc admettre que les activités proposés par Muséo+ ont, à la fois, aidé à baisser le niveau d'excitation que la découverte d'un environnement inconnu et la prise en main de l'application avaient elles-mêmes suscitée et ont également permis une implication dans les activités culturelles, pédagogiques et ludiques. Le tracé global de l'AED (figure5) montre que ces bénéfices n'ont pas été observés dès le début de la séance. A son arrivée et pendant la phase de préparation (explication des consignes, équipement, calibration), l'enfant est dans un état d'excitation extrême. Le niveau global de l'AED est très élevé, de nombreux pics se superposent, le tout étant accompagné de nombreux mouvements du corps (courbe $\mathrm{c}$ de la figure 5). Cette configuration est typique d'un état de stress élevé. 
a

proparation

b

Figure 5 : évolution de l'AED (courbea) du participant 1 sur l'ensemble de la séance.

Les pics constatés sur la courbe a montrent une très forte activité phasique et un niveau d'activation élevé pendant la phase de préparation

(juste avant que la tablette soit proposée). La courbec

(mouvements) montre une très forte agitation.

L'enfant est passé par différentes phases avant de pouvoir profiter de l'application, la figure 6 détaille ces différents moments. Au début (imagea), il a besoin de réconfort et quand il obtient la tablette (image $b$, figure 6), l'excitation est au maximum. La prise en main (images $c$ et $d$ de la figure6) n'est pas aisée mais finalement, l'enfant s'approprie l'outil et s'élance dans les allées du musée (imagese et $f$ ).

a

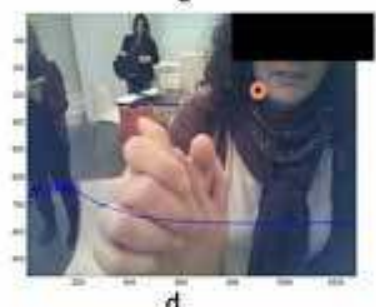

d

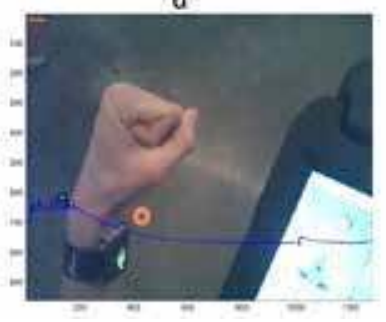

b

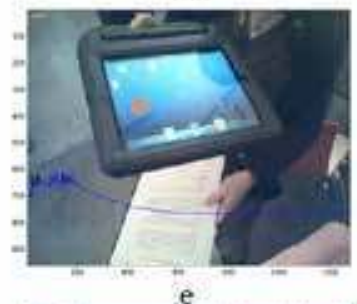

e

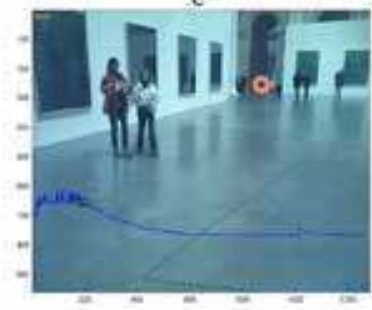

c
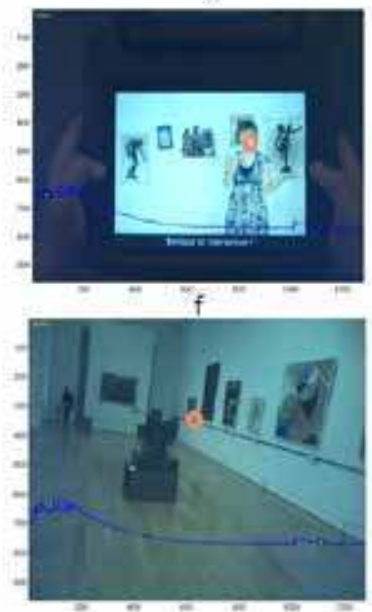

Figure 6: captures d'écran provenant de la caméra de scène du participant 1. Le rond orange indique la position du regard, la courbe bleue représente le tracé de I'AED et le carré vert indique le niveau ponctuel de I'AED au moment de la capture.

En début de séance, l'enfant a besoin d'être réconforté (a), puis la tablette lui est proposée (b) et l'enfant découvre l'environnement proposé par Muséo+. Mais son niveau de stress $(d)$ est encore très élevé. On constate ensuite $(e, f)$ qu'une fois que l'excitation baisse, l'enfant s'élance dans les allées du musée et effectue sans difficulté le parcours proposé. 


\subsubsection{Participant 2}

Il s'agit d'un enfant présenté par ses éducateurs comme "autiste non diagnostiqué». Il ne semble pas souffrir d'une phobie sociale. Ses enregistrements sont très différents du participant précédent. On n'observe pas de phase de stress, au contraire, le niveau global d'activation (niveau tonique, figure 7) est plutôt faible en début de séance mais augmente régulièrement pour finalement se stabiliser à partir de la cinquième séquence. Cette configuration montre un engagement du participant et une implication de plus en plus prononcée dans les activités proposées par l'application. Cet intérêt est confirmé par les réactions phasiques qui montrent que le participant réagit à certaines stimulations provenant de son environnement. En particulier, les séquences 2, 4, 5 et 7 montrent une augmentation des pics d'AED. Mis à part la séquence 5 , ces augmentations d'activité phasique s'accompagnent d'une augmentation de la part d'exploitation. En d'autres termes, cela signifie qu'aux moments où l'enfant se concentre sur les activités proposées par l'application, l'AED augmente. Cette augmentation peut être liée à l'engagement du participant mais peut aussi provenir de réactions affectives puisqu'elles sont corrélées à une montée tonique.

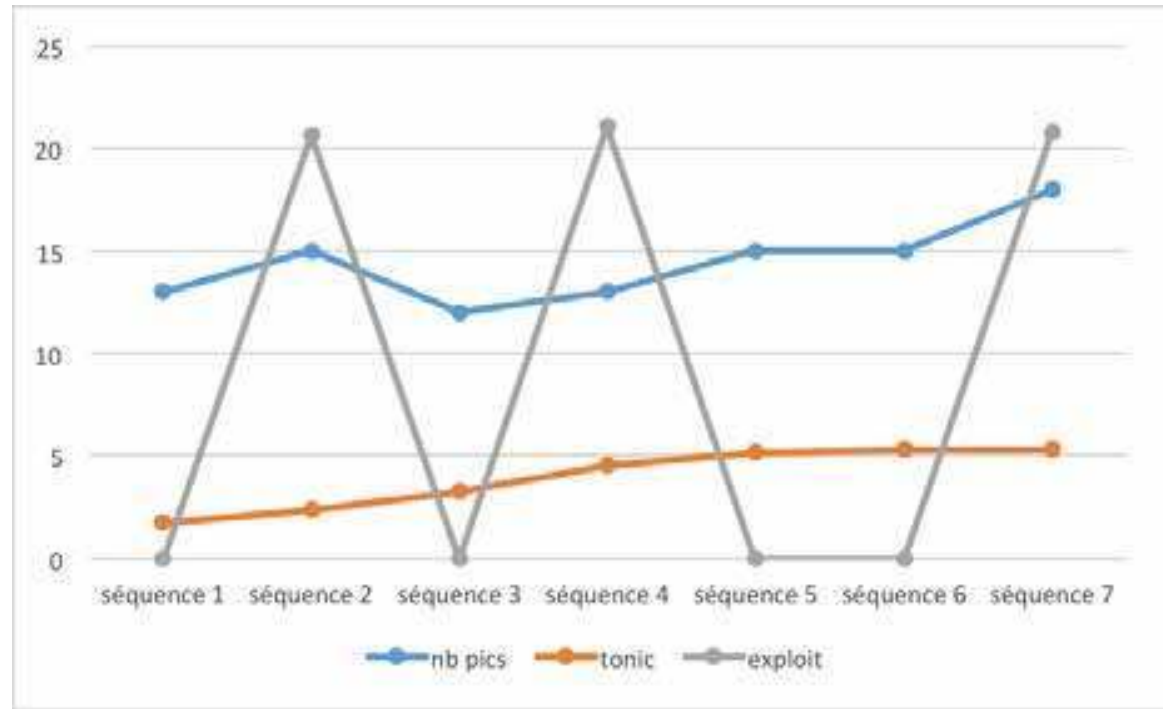

Figure 7: nombre de pics et niveau tonique d'AED.

Part d'exploitation oculaire pour le participant 2

Il est donc clair, à partir de ces données, que les activités proposées par Muséo+ ont bien été acceptées par le participant, même si le niveau d'engagement n'est pas forcément toujours stable et que certaines activités l'intéressent plus que d'autres. Les augmentations synchronisées de la part d'exploitation, de la tonicité et des réactions phasiques montrent clairement que les activités 
proposées sont bien acceptées et que, très probablement, elles génèrent des réactions affectives plutôt positives. En tout cas, la configuration obtenue est très différente de celle du participant 1 (diagnostiqué comme autiste), ce qui laisse planer un doute sur la prise en charge actuelle de ce jeune, considéré à I'heure actuelle comme autiste.

\subsubsection{Participant 3}

Le participant $n^{\circ} 3$ n'a pas de problème particulier de développement. On observe (figure 8) que cet enfant réalise peu d'exploitation sauf lors de la séquence 5. Cela signifie que cette partie a mobilisé son attention plus que pour les autres séquences, mais cela ne signifie pas pour autant que les autres activités ne l'ont pas intéressé.

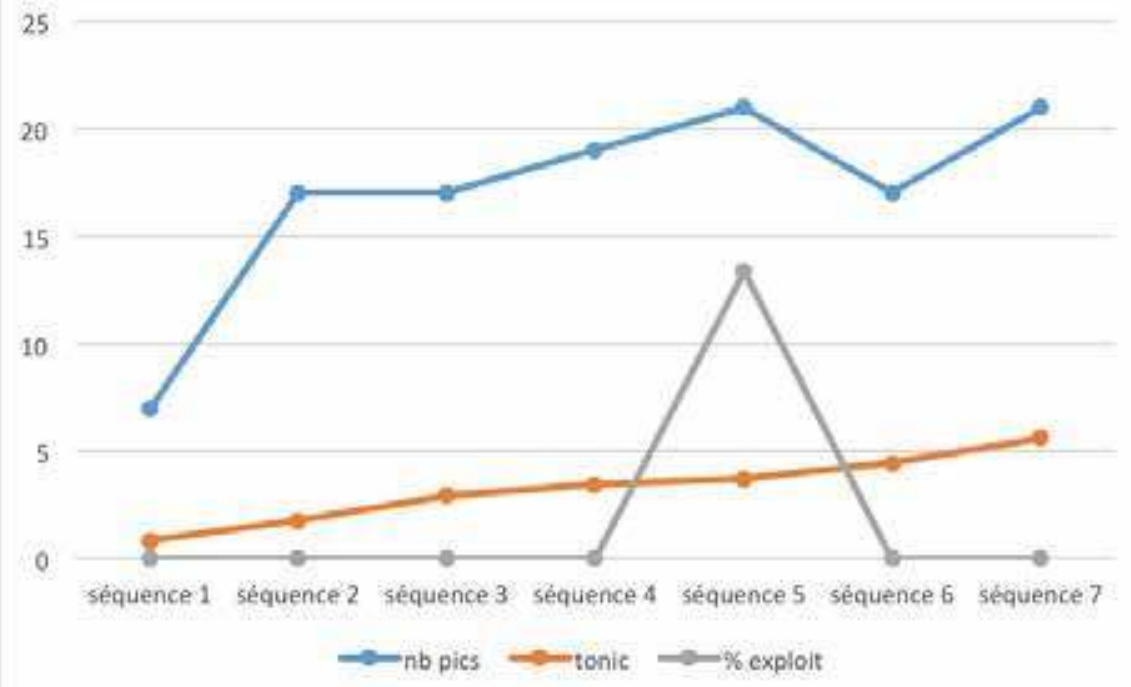

Figure 8: nombre de pics et niveau tonique de I'AED.

Part d'exploitation oculaire pour le participant 3

En effet, d'une part, la classification des déplacements oculaires en exploration/exploitation est plus difficile pour ce participant étant donné qu'il réalise des déplacements rapides et fréquents et que le nombre de clignements est un peu plus important. Cette configuration rend plus difficile la classification entre exploration (grandes saccades suivies de fixations de durée plus longue) et exploitation (petites saccades suivies de fixations plus courtes) car moins de données sont disponibles. D'autre part, on constate aussi (voir figure9) que ce participant réalise des séquences d'exploitation surtout pour les moments où le plan du musée est consulté afin de découvrir le chemin menant à l'œuvre. 


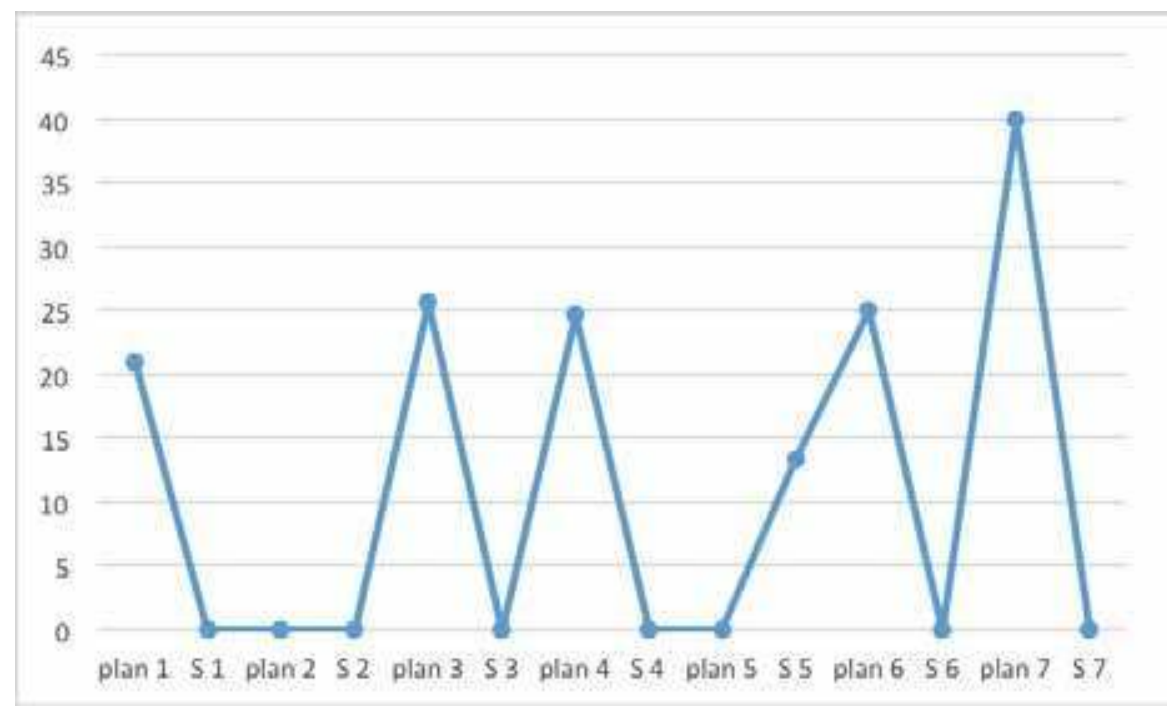

Figure 9: part d'exploitation oculaire pour le participant 3 pour les parties "plan» (consultation du plan du musée afin de découvrir le chemin menant à l'œuvre) et pour les séquences de découverte de l'œuvre (S1à S7)

Ce comportement n'est pas observé pour les autres participants (tableau2): en général, la consultation du plan entraîne de nombreux déplacements oculaires (en particulier, de guidage des déplacements moteurs) qui sont difficiles à classer en exploration/exploitation puisque les participants sont en mouvement. Cette configuration atypique obtenue pour le participant 3 peut signifier qu'il a besoin de plus de ressources attentionnelles pour bien comprendre le plan et se repérer dans l'environnement qui l'entoure.

\begin{tabular}{ccccc} 
& $\mathrm{P} 1$ & $\mathrm{P} 2$ & $\mathrm{P} 3$ & $\mathrm{P} 4$ \\
\hline plan 1 & 0,00 & 0,00 & 20,91 & 0,00 \\
plan 2 & 0,00 & 0,00 & 0,00 & 0,00 \\
plan 3 & 0,00 & 27,27 & 25,68 & 0,00 \\
plan 4 & 0,00 & 0,00 & 24,69 & 0,00 \\
plan 5 & 0,00 & 30,00 & 0,00 & 0,00 \\
plan 6 & 0,00 & 0,00 & 25,07 & 0,00 \\
plan 7 & 9,23 & 0,00 & 40,00 & 0,00 \\
\hline
\end{tabular}


Un autre élément mettant en évidence le fait que le parcours est bien réalisé et mobilise le participant provient de l'analyse de I'AED. On constate une montée régulière de la tonicité et des réactions phasiques en constante augmentation, en particulier pour la séquence 5 où le pic d'activité phasique correspond aussi à un pic d'activité d'exploration, ce qui confirme bien que cette séquence a particulièrement mobilisé les ressources du participant, mais que les autres ne l'ont pas non plus désintéressé.

\subsubsection{Participant 4}

Le quatrième participant n'a pas de problème de développement particulier. On observe une augmentation régulière de la part d'exploitation (figure10) avec un pic pour la séquence 6 . De façon concomitante, l'activité phasique est aussi à son maximum pour cette séquence, mais on note aussi une très forte augmentation de l'activité phasique à partir de la cinquième séquence. Le niveau tonique progresse aussi régulièrement à partir de cette même séquence. Cette configuration montre une très forte implication de ce participant ainsi qu'un intérêt très marqué pour les activités proposées et qui apparaît vers le milieu du test. Un premier pic phasique modéré apparait dès la séquence 3 et les niveaux d'exploitation, de réponses phasiques et toniques ne cessent d'augmenter à partir de ce moment-là.

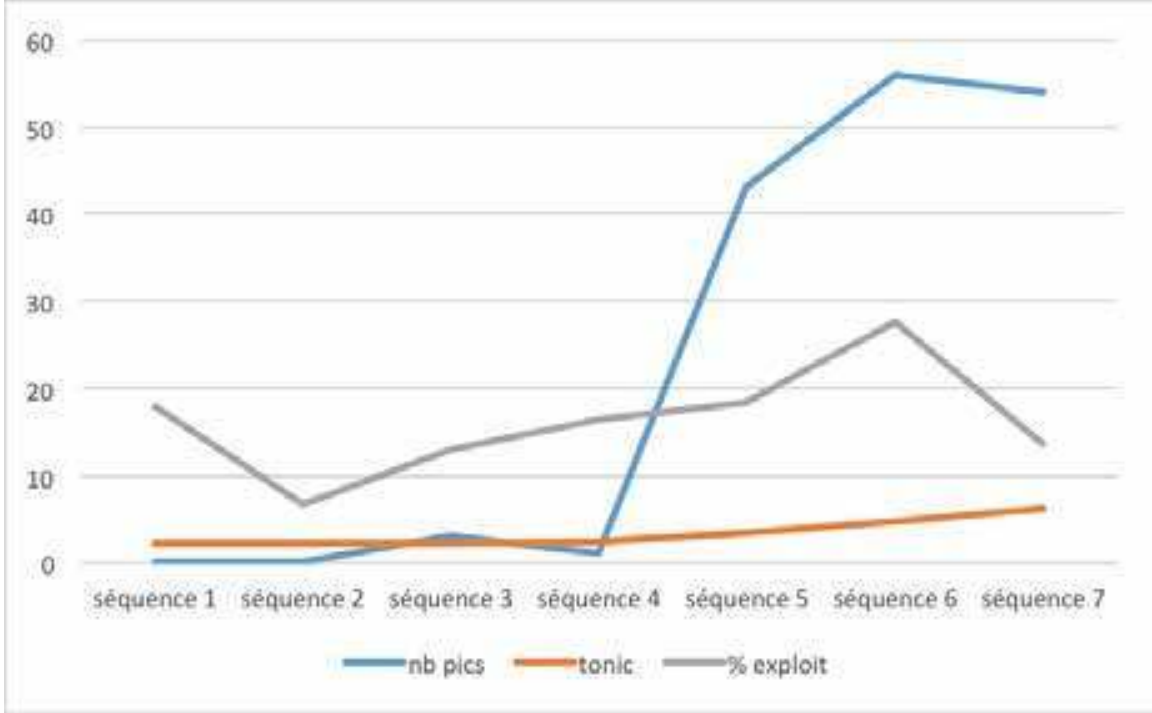

Figure 10: nombre de pics et niveau tonique de l'AED. Part d'exploitation oculaire pour le participant 4 


\subsubsection{Conclusion partielle}

Cette démarche qui associe l'enregistrement des déplacements oculaires et l'enregistrement de l'AED en ambulatoire lors d'une visite au musée s'avère particulièrement intéressante. Des points communs (concentration de l'attention visuelle associée à des changements de l'AED) sont observés entre les participants mais aussi des particularités permettant de mieux comprendre les effets que provoque l'application Muséo+ sur le comportement. Tous les participants sont équipés dès leur arrivée et l'enregistrement commence bien avant la remise de la tablette. Le participant1, autiste, est en sur-stress maximal dès le début. Dans son cas, I'application permet de baisser le niveau d'activation ou de stress, ce qui favorise une utilisation efficace de la tablette. Dans le cas du participant 2 , on observe que les activités proposées peuvent parfois générer des niveaux de concentration plus élevés (les séquences 2, 4 et 7) mais toujours accompagnés de réponses physiologiques mettant en évidence des interactions accrues avec l'environnement dans lequel l'observateur est placé. Enfin, pour d'autres, (participants 3 et 4), la prise d'informations liées aux séquences pédagogiques peut être plus variable ou plus concentrée sur une activité, mais à chaque fois, les indicateurs physiologiques montrent un niveau d'engagement en constante augmentation.

Par conséquent, cette méthodologie permet de mettre en évidence que, pour ces utilisateurs pour lesquels le test est concluant, l'application Muséo+ est parfaitement bien prise en main et qu'elle génère un intérêt marqué, qui en outre va généralement croissant. Toutefois, il convient de souligner combien les effets observés sont variés. La méthode permet de mesurer concrètement à quel point les comportements et réactions sont individualisés, et ne peuvent nullement se transférer d'un participant à un autre. Ce résultat met en exergue les limites des opérations statistiques, qui moyennent des expériences sans rapport les unes avec les autres.

\section{3. \\ Mesure motivationnelle par le diagramme valence-activation}

\subsection{Méthodologie}

Des méthodes plus classiques, de type questionnaires, ont également été mobilisées non pas auprès des enfants eux-mêmes mais auprès de leurs accompagnants. En parallèle, le recours à une forme de mesure non verbale a là encore été privilégié. II leur a ainsi été demandé de positionner l'état de l'enfant au début et en fin de visite dans le plan du diagramme valence-activation (Lang, 1994) qui repose sur des paramètres motivationnels définissant une disposition générale à éviter ou à approcher une stimulation et sa vigueur (Lang et al., 1993): ils caractérisent donc l'évaluation émotionnelle d'un vaste ensemble de stimuli 
perceptifs et symboliques (Bradley et Lang, 1994). Ces deux indicateurs sont côtés à l'aide de deux axes hérités des échelles du Self-Assessment Manikin (SAM), dont le modèle a été mis au point par Bradley et Lang (1994). Chacun de ces axes porte en ses extrémités deux émoticônes schématisés par des expressions faciales. Pour la valence-i.e. appréciation négative versus positive-, on passe d'un personnage au visage grognon à un personnage souriant. L'axe activation débute par un personnage sous-activé, c'est-à-dire relaxé, voire endormi, pour aboutir à un personnage en activation maximale, qui paraît excité, énervé. Pour chaque évaluation, les enfants se positionnent seuls ou avec l'aide des accompagnants (les parents ou les éducateurs) en fonction des deux axes. Pointons cependant le fait que ce modèle permet uniquement de relever une mesure déclarative de l'émotion. Toutefois, plusieurs recherches (Cheesman et Merikle, 1986; Kunimoto et al., 2001; Merikle et Daneman, 2000; Merikle et al., 2001; Ohman, 1999) soulignent sa pertinence et proposent d'y associer des mesures telles que les mesures physiologiques oculométriques et électrodermales, ce qui est le cas dans notre étude.

Un point reflétant l'état de l'enfant est ainsi positionné dans le plan au début et en fin de visite de manière à caractériser et à pouvoir quantifier la progression de cet état dans l'espace de valence-activation sous l'effet de l'expérience de visite. La position du point dans le plan constitue une deuxième forme, cette fois déclarative mais toujours non verbale, de mesure de l'appétence-intéressement complémentaire aux relevés d'eye-tracking.
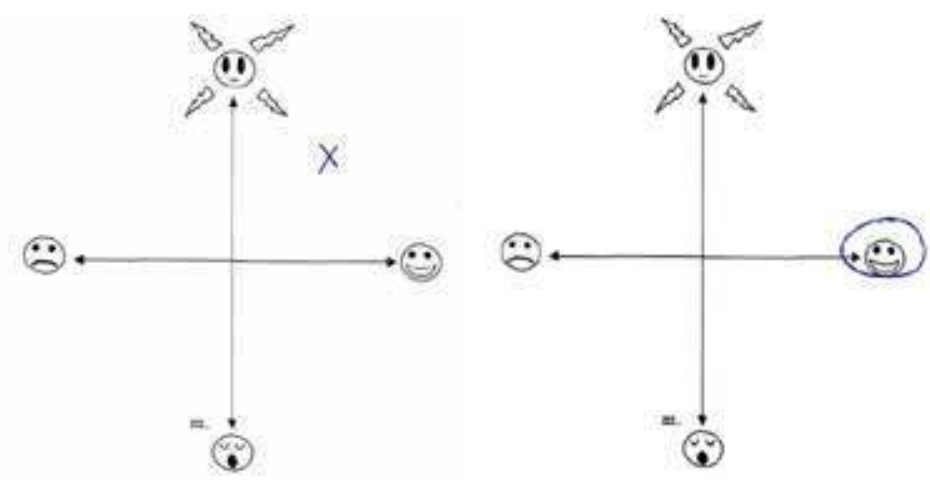

Figure 11: diagramme valence-activation complété avant et après la visite: le cas de Ed, participant 1 de l'expérience précédente

\subsection{Résultats}

Le recours à ce diagramme pour les 130 enfants démontre que l'état des enfants autistesa, pour tous, évolué vers plus de calme et plus d'intérêt, soit en direction du quart inférieur droit. Notons que, pour ces publics particuliers, une baisse de l'activation ne signifie pas un désengagement, mais un apaise- 
ment. C'est donc un bénéfice, au contraire des lectures habituelles. Celui-ci est corroboré par les entretiens, où certains parents ont dit par exemple qu'ils «n'auraient jamais pensé pouvoir passer un moment aussi serein au musée avec leur enfant autiste». La baisse du stress s'accompagne, dans la majorité des cas, d'une hausse de la concentration et de la réactivité aux consignes, ce que tend à corroborer l'analyse des données statistiques de l'application. De même, les stéréotypies, ou encore l'écholalie ${ }^{5}$, diminuent de manière significative quand elles sont avérées. L'attention est donc bien focalisée sur l'application et l'importance accordée aux facteurs de stress générés par cette visite ponctuelle est reléguée au second plan, ou s'efface momentanément, au profit de l'engagement dans la visite.

Pour les enfants sans problème de développement particulier, l'activation augmente et la valence évolue vers le positif, soit une évolution en sens inverse des cas d'autisme. Comme pour les mesures oculométriques et d'AED, l'outil de mesure met en évidence les différences comportementales entre enfants autistes et enfants sans handicap - comme l'avait déjà montré la comparaison entre les tracés des participants 1 et 2 dans l'expérimentation précédente.
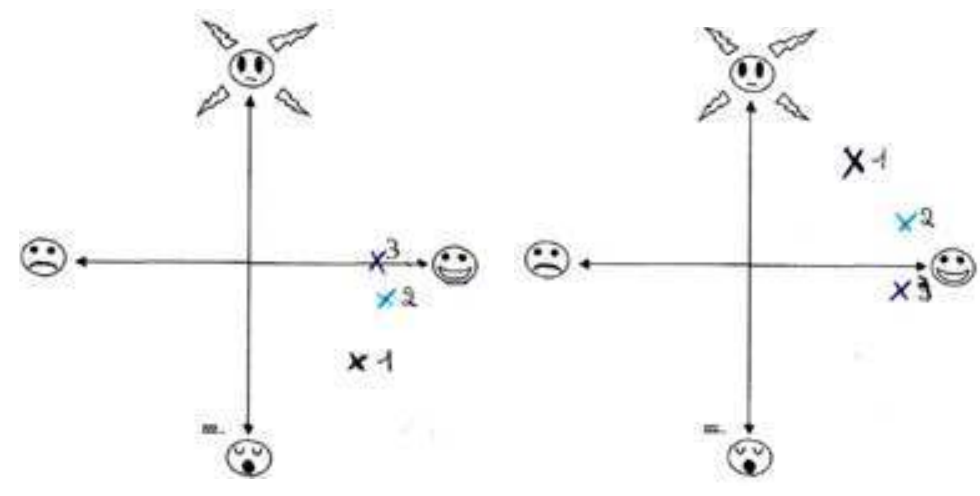

Figure 12: diagramme valence-activation complété avant et après la visite: les cas de Ma(1), Sy(2) et To(3) sans handicap: la valence et/ou l'activation restent stables ou augmentent

5 Les stéréotypies et l'écholalie-cris, mouvements saccadés, répétitions ad infinitum...- sont les manifestations physiques et comportementales du surstress chez l'autiste. Elles sont directement observables sans appareillage ni protocole particulier. 


\section{4. Conclusion}

Dans cette première étude exploratoire, l'échantillon soumis aux mesures physiologiques est très limité (4enfants). En revanche, les mesures émotionnelles par valence-activation, moins lourdes en termes de protocole, ont été effectuées pour les 130 enfants. Une démarche poppérienne falsificationniste a été adoptée: elle suppose que les résultats de l'une et l'autre méthode convergent. Les tests empiriques ont confirmé cette hypothèse. La future conduite d'autres expériences similaires augmentera progressivement la probabilité de sa véracité, jusqu'à une éventuelle falsification. Suivant une telle approche scientifique, en aucun cas l'augmentation de l'échantillon ne démontrera la propriété: il ne fera qu'augmenter toujours plus la probabilité de sa véracité.

Partant de cette hypothèse, les deux méthodes convergent vers les résultats suivants: dans le cadre spécifique qui était le nôtre et pour les enfants testés, ils semblent attester le double effet captivant et apaisant de la médiation par tablette au musée pour les enfants atteints de déficit intellectuel ou d'autisme. Dans le cas de Muséo+, la tablette a permis une médiation communicationnelle entre les enfants et les œuvres d'art, pour un public auprès duquel la médiation usuelle, humaine et/ou verbale, est rejetée. Non seulement la visite est rendue possible alors qu'elle ne l'était pas auparavant (manifestations de sur-stress, stéréotypies, écholalies, rejet, etc.), mais l'enfant l'effectue en complète autonomie. L'ensemble des relevés témoigne en outre de son engagement dans l'activité, de sa concentration, et d'appréciations positives. Pour les enfants sans problème de développement particulier, les relevés sont foncièrement différents, mais ils témoignent également d'un engagement dans l'activité, d'une augmentation de la concentration et d'appréciations positives. La dimension «universelle»- au sens du Design for all-du dispositif est ainsi démontrée.

En parallèle, le résultat le plus frappant est la diversité des effets observés. En effet, chaque «séquence» des relevés (figures 3, 4, 7, 8 et 10) correspond à une œuvre. La méthode montre que la question «quelle est l'œuvre qui fonctionne le mieux? " n'est pas pertinente. En effet, tel enfant concentrera ses ressources cognitives sur telle œuvre, mais tel autre enfant ressentira une vive émotion avec une autre, tandis qu'un troisième sera plus égal et plus distribué dans ses réactions et ses comportements. Cette observation disqualifie tout traitement statistique pour analyser les modes d'appropriation des œuvres. Elle montre au contraire l'intérêt d'une recherche située au niveau de l'individu.

Enfin, les résultats obtenus doivent amener le professionnel de musée à intégrer que, pour certains publics, l'accessibilité aux œuvres peut dans certains cas être favorisée par un dispositif de médiation numérique bien conçu. Ils conduisent aussi à prendre toute la mesure de la diversité des expériences de visite, et à en tenir pleinement compte dans une approche renouvelée de sa programmation et de sa stratégie. 
Bibliographie

Boucsein W. (2012). Electro-

dermal activity, Springer.

BougeniesF., Houriez S., Houriez J. et Leleu-MervielS. (2015). "Musée pour tous. Un dispositif de découverte dans les murs et son évaluation ". In Culture \& Musées $n^{\circ} 26$, "Culture dans/hors les murs et publics atypiques», pp.115-139.

Bradley M. M. et Lang P. J. (1994). "Measuring emotion: The Self Assessment Manikin and the Semantic Differential». In Journal of Behavior Therapy and Experimental Psychiatric, 25, pp.49-59.

Cheesman J. et Merikle P. M. (1986). "Distinguishing conscious from unconscious perceptual processes ». In Canadian Journal of Psychology, ${ }^{\circ} 40$, pp.343-367.

Gilzenrat M.S., Nieuwenhuis S., Jepma M. et Cohen J.D. (2010). «Pupil diameter tracks changes in control state predicted by the adaptive gain theory of locus coeruleus function". In Cognitive, Affective, \& Behavioral Neuroscience, $\mathrm{n}^{\circ} 10$ (2), pp.252-269.

Houriez S., Houriez J., Kounakou K. et Leleu-MervielS. (2013). «Accessibilité des musées: de la conception pour les enfants sourds au design for all ». In MEI n³6, pp. 25-37, Numéro Handicap et Communication.
Kounakou K. et VerclytteL. (2011). "VI.A.G.E.: un protocole pour cerner l'impact des images médiatiques sur la petite enfance». In RIHM: Revue des Interactions Humaines Médiatisées, $\mathrm{n}^{\circ} 12(1)$, pp.63-100.

Kunimoto C., Miller J. et Pashler H. (2001). "Confidence and accuracy of near-treshold discrimination responses». In Consciousness and Cognition, n¹0, pp.294-340.

Land M., Mennie N. et Rusted J. (1999). «The roles of vision and eye movements in the control of activities of daily living ". In Perception, $n^{\circ} 28(11)$, pp.1311-1328.

Lang P. J. (1994). «The motivational organization of emotion: affect-reflex connections ». In VanGoozen S., Van de Poll N.E. and Sergeant J.A. (Eds.), Emotions: Essays on Emotion Theory, Erlbaum, Hillsdale, NJ, pp.61-93.

Lang P. J., Greenwald M. K., Bradley N. M. et Hamm A. O. (1993). «Looking at pictures: Affective, facial, visceral, and behavioral reactions ". In Psychophysiology, $\mathrm{n}^{\circ}$ 30, pp.261-273.

Leleu-MervielS. et Kounakou K. (2011). «Muséo: un visio-guide interactif à l'épreuve». In RIHM: Revue des Interactions Humaines Médiatisées, n¹2(2), pp.25-65.

MerikleP.M. et Daneman M. (2000). "Conscious vs. Unconscious pereption». In M.S. Gazzanig (dir.), The new cognitive neurosciences, Cambridge, MA, MIT Press, pp.1295-1303. 
MerikleP.M., SmilekD. et

Eastwood J.D. (2001). «Perception without awareness: Perspectives from cognitive psychology». In Cognition, $n^{\circ} 79$, pp.115-134.

Ohman A. (1999). «Distinguishning unconscious from conscious emotional processes: Methodological considerations and theorical implications ». In T. Dalgleish \& M. Power (dirs.), Handbook of cognition and emotion, Chichester, U.K., Wiley, pp.321-352.

Papesh M.H., Goldinger S. D. et Hout M.C. (2012). «Memory strength and specificity revealed by pupillometry ». In International Journal of Psychophysiology: Official Journal of the International Organization of Psychophysiology, n83(1), pp.56-64.

Reinhardt T., SchmahlC., Wüst S. et Bohus M. (2012). «Salivary cortisol, heart rate, electrodermal activity and subjective stress responses to the Mannheim Multicomponent Stress Test (MMST)». In Psychiatry Research, n¹98(1), pp.106-111.

Steinhauer S.R., Siegle G. J., Condray R. et Pless M. (2004). «Sympathetic and parasympathetic innervation of pupillary dilation during sustained processing ". In International Journal of Psychophysiology, n52(1), pp.77-86. 